\title{
Transmissor QPSK em Banda X para Aplicação Espacial
}

\author{
Ivan Tosetto, Lucio Cividanes, Bruno Santos, Luciano Silva e Rodolfo Araújo \\ Instituto Nacional de Pesquisas Espaciais - INPE, São José dos Campos, SP, 12245-970, Brasil
}

\begin{abstract}
Resumo - Este trabalho apresenta o projeto e os detalhes de construção do modelo de desenvolvimento de um transmissor QPSK na banda X para aplicação no Subsistema de Transmissor de Dados (MWT) das câmeras WFI e MUXCAM dos Satélites CBERS 3\&4 operando com taxas de transmissão de dados de 53 Mbps e 68 Mbps respectivamente. Os resultados dos testes apresentados confirmam o bom desempenho do equipamento.
\end{abstract}

Palavras-Chave - banda X, transmissor QPSK, aplicação espacial, CBERS 3\&4.

Abstract - This work presents the design and construction details of a QPSK transmitter development model in $X$ Band for application in the Data Transmitter Subsystem (MWT) of the CBERS 3\&4 satellite cameras WFI and MUX which respectively operate with 53 Mbps and 68 Mbps transmission rates. The test results validate the equipment performance.

Index Terms -X Band, QPSK transmitter, space application, CBERS 3\&4.

\section{INTRODUÇÃO}

O Instituto Nacional de Pesquisas Espaciais - INPE em cooperação com a Academia Chinesa de Tecnologia Espacial - CAST - vem trabalhando para a construção dos satélites de sensoriamento remoto CBERS $3 \& 4$ [1]. Estes satélites são compostos de quatro câmaras de imageamento, sendo duas de responsabilidade brasileira: MUX - MultiEspectral que opera com taxa de dados de $68 \mathrm{Mbits} / \mathrm{s}$ e o WFI - Imageador de Campo Largo que opera com taxas de $53 \mathrm{Mbits} / \mathrm{s}$ [2].

Para a modulação / transmissão dos dados provenientes destas câmaras, um transmissor QPSK em banda X foi projetado utilizando componentes modulares com o intuito de otimizar massa, volume e potência.

Convém ressaltar que transmissores em banda $\mathrm{X}$, além de possuírem altíssimo custo, são encontrados somente no mercado internacional, com restrições de importação impostas pelo ITAR (International Traffic in Arms Regulations [3]). Daí, a principal motivação do desenvolvimento desse equipamento.

Ivan Tosetto, Lucio Cividanes, Bruno Santos, Luciano Silva e Rodolfo Araújo. Divisão de Eletrônica Aeroespacial (DEA), Instituto Nacional de Pesquisas Espaciais (INPE), São José dos Campos, Brasil, E-mails: ivan@dea.inpe.br, lucio@dea.inpe.br,bruno@dea.inpe.br ,luciano@dea.inpe.br e rodolfo@dea.inpe.br

\section{DESCRIÇÃO DO TRANSMISSOR (TX)}

A foto do transmissor em bancada pronto para realização dos testes é apresentado na Figura 1 e na Figura 2, é mostrado o diagrama em blocos.

O equipamento foi projetado para receber os dados provenientes das câmaras, modulá-los, amplificá-los e transmiti-los para uma Estação Terrena (no caso do TX embarcado). O transmissor está dividido em três partes tal como descrito abaixo:

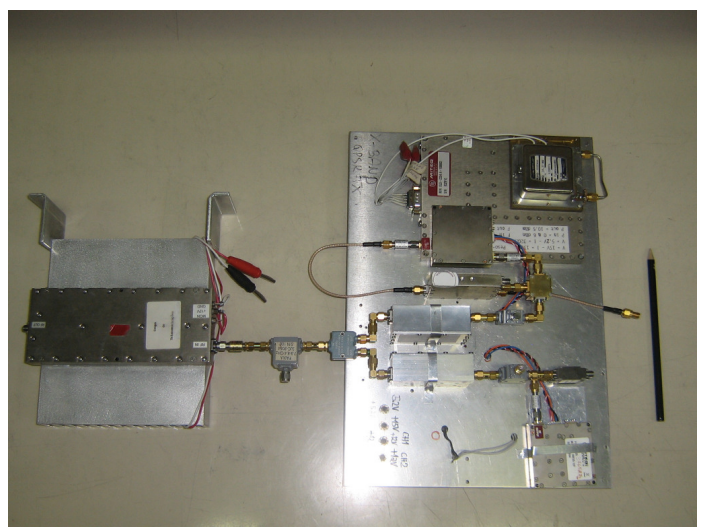

Fig. 1. Foto do Transmissor, mostrando à esquerda o SSPA

\section{A - Canal WFI:}

Este canal tem seus sinais modulantes (I e Q), com taxas de 26,5 Mbits/s provenientes da câmara WFI, aplicados ao modulador QPSK (MITEQ modelo SDM0104LC1MD [4]) em banda $\mathrm{S}(2204,4 \mathrm{MHz})$ primeiramente, sendo deslocados logo em seguida para banda X $(8290 \mathrm{MHz})$ através de um Up Converter. O TCXO $(18,37 \mathrm{MHz})$ fornece a referência externa com alta estabilidade ao sintetizador (MITEQ série SLS [4]) (2204,4 MHz) gerando assim a portadora em banda $\mathrm{S}$ com grande precisão e baixo ruído de fase $\left(0,21^{\circ}\right.$ integrado de $100 \mathrm{~Hz}$ a $1 \mathrm{MHz}$, medido no analisador de sinais Rohde\&Schwarz modelo FSQ 26). O ruído de fase é um requisito importante para um transmissor com modulação QPSK [5].

São utilizados atenuadores $(10 \mathrm{~dB})$ na entrada e na saída (6 dB) do modulador para adequar ao nível correto dos circuitos e melhorar o casamento entre eles.

Em seguida, o sinal modulado é deslocado para a banda $\mathrm{X}$ (8289,4 MHz) através do batimento com o sinal do sintetizador em banda C (6085 MHz). 
A cadeia (WFI) termina com um filtro passa faixas $(8290$ +/- $800 \mathrm{MHz}$ ) que garante a eliminação de harmônicos.

\section{B - Canal MUX:}

Este canal tem seus sinais modulantes (I e Q), provenientes da câmara MUX com taxas de $34 \mathrm{Mbits} / \mathrm{s}$, enviados diretamente ao modulador QPSK (MITEQ modelo SDM0708LI3CDQ [4]) em banda X, eliminando dessa maneira o Up-Converter como no caso do Canal WFI. A portadora em banda $\mathrm{X}$ é gerada a partir de um sintetizador de frequiências (MITEQ série SLS [4]) com baixo ruído de fase $\left(0,8^{\circ}\right.$ integrado de $100 \mathrm{~Hz}$ a $1 \mathrm{MHz}$, medido no analisador de sinais Rohde\&Schwarz modelo FSQ 26).

O nível de cada sinal I e Q foi ajustado para se obter um sinal modulado na saída com aproximadamente a mesma potência do sinal de saída do canal WFI.

Novamente, a cadeia (MUX) termina com um filtro passa faixas $(8365+/-800 \mathrm{MHz})$ garantindo assim a eliminação de harmônicos.

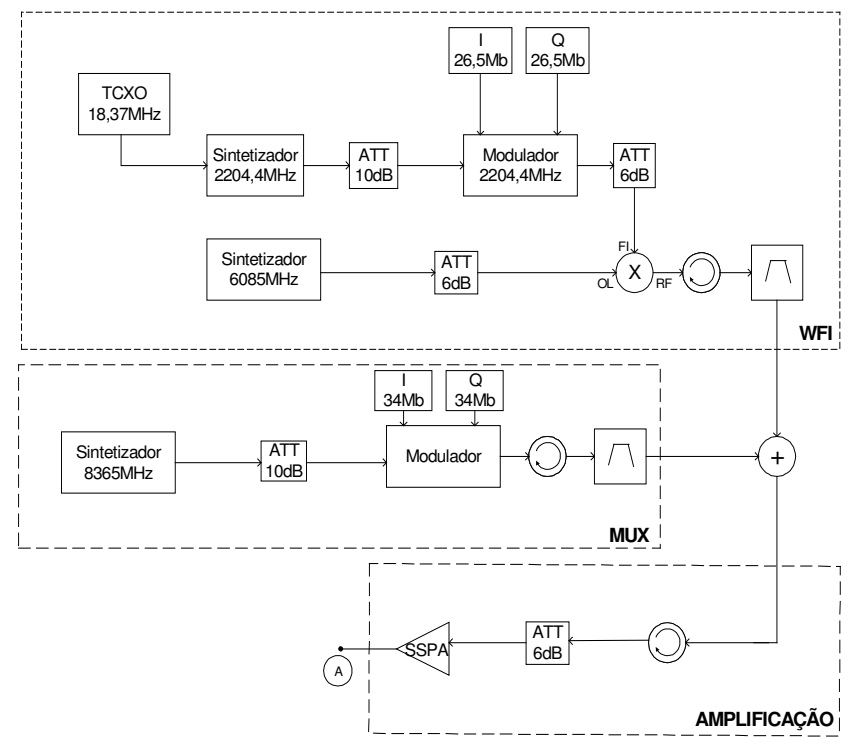

Fig. 2. Diagrama de blocos do transmissor.

\section{C-Amplificação do sinal}

Os sinais provenientes dos canais WFI e MUX (ambos em banda $X)$ são então somados e amplificados por um amplificador de estado sólido SSPA da FUJITSU com ganho de $57 \mathrm{~dB}$, fornecendo na saída $4 \mathrm{~W}$ de potência RF.

Como pode ser observado pelo diagrama (Fig. 2), após o somador o sinal passa por um circulador garantindo um melhor casamento com a entrada do SSPA. Um atenuador de $6 \mathrm{~dB}$ é utilizado para se ajustar o nível do sinal de entrada do amplificador para operação próximo ao ponto de compressão de $1 \mathrm{~dB}$.
Para fechar o sistema de comunicações e para a realização dos testes de BER (bit error rate), o sinal composto passa por um Down Converter caindo no centro da faixa (720 $\mathrm{MHz}$ ) de demodulação do receptor IN-SNEC [6]. O filtro passa baixas $(\mathrm{Fc}=2 \mathrm{GHz})$ elimina os harmônicos decorrentes do Down Converter. Por fim, adiciona-se ruído ao sinal de maneira a se obter a relação $\mathrm{E}_{\mathrm{b}} / \mathrm{N}_{0}(10,5 \mathrm{~dB})$ conhecida para medir a taxa de erro de bit e a degradação de cada canal (Figura 3).

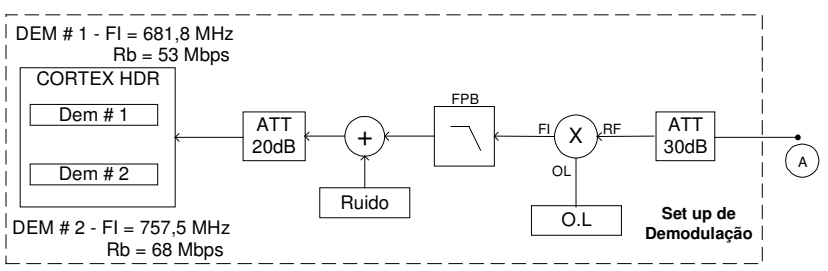

Fig. 3. Sistema de recepção utilizado para demodulação

\section{RESULTADOS OBTIDOS}

Esta seção apresenta alguns dos resultados obtidos com o transmissor.

A Fig. 4 mostra o espectro medido do sinal WFI com modulação QPSK em banda X (8290 MHz).

Nota-se que o sinal encontra-se bem balanceado, com uma diferença de aproximadamente $13 \mathrm{~dB}$ entre o nível da portadora principal e os lóbulos laterais [7].

Na Fig. 5 é mostrado o espectro QPSK medido da câmara MUX. Para a realização dessas medidas, foi utilizado o Analizador de Sinais Rohde\&Schwarz FSQ26 e o sistema de recepção mostrado na Fig. 3. Neste esquema, as portadoras dos canais WFI e MUX, após o down converter, encontramse em $681,8 \mathrm{MHz}$ e 757,5 MHz respectivamente.

A Fig. 6 apresenta a sobreposição dos espectros dos dois canais, de modo a visualizar a possível interferência entre os dois canais. Apesar de a interferência estar abaixo do nível de ruído, podemos observar os lóbulos laterais do canal 1 invadindo o lóbulo principal do canal 2 e vice versa, confirmando a existência da interferência entre os dois canais.

Para minimizar a degradação por interferência entre os canais, é necessária a implementação de uma formatação dos dados na transmissão (por exemplo, implementando um filtro tipo cosseno levantado) ou a inclusão de um filtro de RF (OMUX - Output Multiplexer) tipo elíptico na saída do transmissor, como previsto no projeto MWT [2]. 


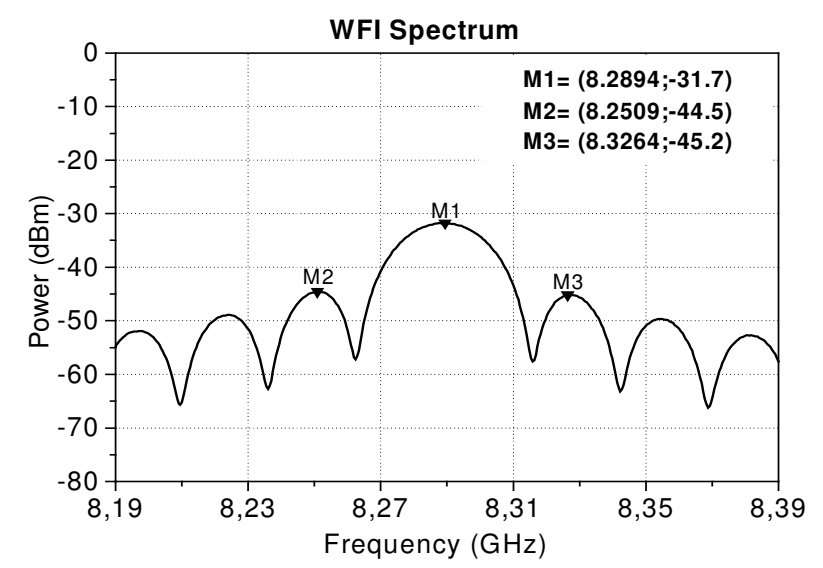

Fig. 4. Espectro do sinal WFI modulado QPSK em banda X.

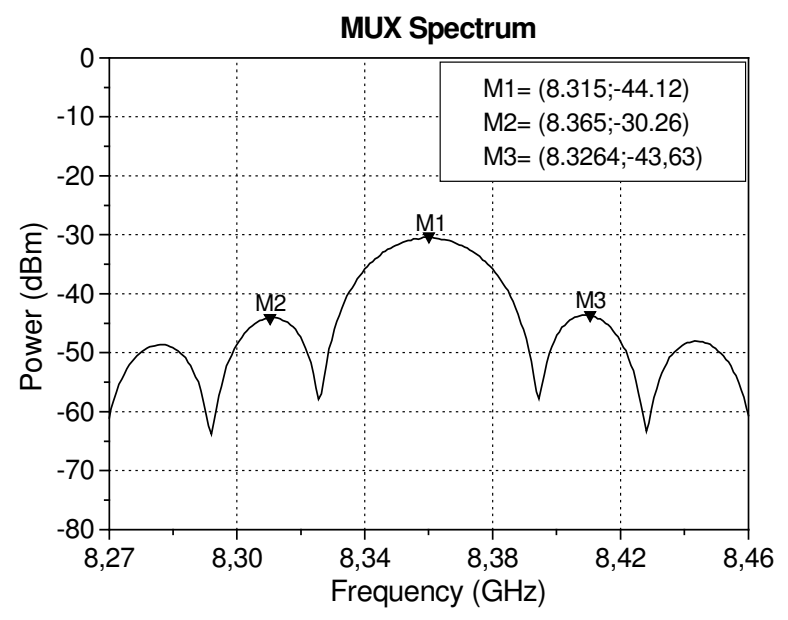

Fig. 5. Espectro do Sinal MUX modulado QPSK em banda X.

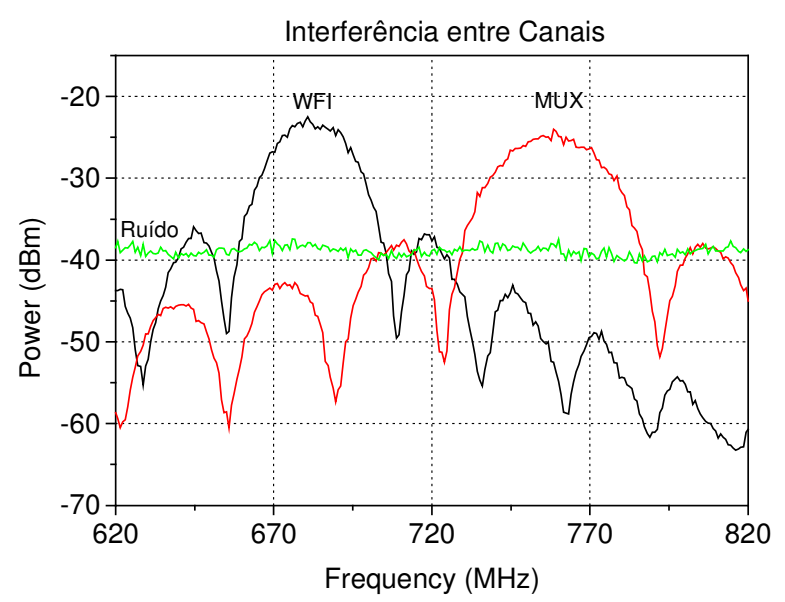

Fig. 6. Espectros sobrepostos na entrada do receptor.
As medidas realizadas (Tabelas I) foram comparadas com os valores especificados para o Subsistema MWT [2].

TABELA I

Especificações X Resultados

\begin{tabular}{|c|c|c|c|}
\hline PARAMETROS & ESPEC. & $\begin{array}{l}\text { CANAL } \\
\text { MUX }\end{array}$ & $\begin{array}{l}\text { CANAL } \\
\text { WFI }\end{array}$ \\
\hline $\begin{array}{l}\text { Potencia de saída (Pin } \\
=-21 \mathrm{dBm} \text { ) }\end{array}$ & $33 \mathrm{dBm}$ & $\begin{array}{l}33,6 \\
\mathrm{dBm}\end{array}$ & $33,8 \mathrm{dBm}$ \\
\hline $\begin{array}{l}\text { Degradação de Eb/N0 } \\
\text { no canal (exceto por } \\
\text { interferência) }\end{array}$ & $\leq 6\left(^{*}\right)$ & $0,6 \mathrm{~dB}$ & $0,4 \mathrm{~dB}$ \\
\hline $\begin{array}{l}\text { Degradação por } \\
\text { interferência }\end{array}$ & $\leq 6\left(^{*}\right)$ & $2,9 \mathrm{~dB}$ & $3,2 \mathrm{~dB}$ \\
\hline Isolação entre canais & $\geq 40 \mathrm{~dB}$ & $28 \mathrm{~dB}$ & $21 \mathrm{~dB}$ \\
\hline $\begin{array}{l}\text { Desbalanceamento de } \\
\text { Fase }\end{array}$ & \pm 30 & $1,4^{\circ}$ & $2,38^{\circ}$ \\
\hline $\begin{array}{l}\text { Desbalanceamento de } \\
\text { Amplitude }\end{array}$ & $1 \mathrm{~dB}$ & $1 \mathrm{~dB}$ & $0,2 \mathrm{~dB}$ \\
\hline EVM & $8 \%$ & $6,2 \%$ & $2,7 \%$ \\
\hline $\begin{array}{l}\text { Ruído de Fase (de } 100 \\
\text { Hz a } 1 \mathrm{MHz} \text { ) }\end{array}$ & $10^{\circ} \mathrm{rms}$. & $\begin{array}{l}0,8^{\circ} \\
\text { rms. }\end{array}$ & $1^{\circ} \mathrm{rms}$. \\
\hline Harmônicos & $<40 \mathrm{dBc}$. & \multicolumn{2}{|c|}{$<-60 \mathrm{dBc}$} \\
\hline Espúrios & $<50 \mathrm{dBc}$. & \multicolumn{2}{|c|}{$<-60 \mathrm{dBc}$} \\
\hline SSPA P1dB & $36 \mathrm{dBm}$ & \multicolumn{2}{|c|}{$35,9 \mathrm{dBm}$} \\
\hline
\end{tabular}

(*) A degradação especificada refere-se ao valor total para o canal, incluindo interferência.

(**) A especificação de isolação diz respeito ao valor total incluindo o filtro do OMUX, que é considerado um equipamento à parte do transmissor.

É interessante notar que o baixo valor medido para o ruído de fase do sinal em banda $\mathrm{X}$ indica o bom desempenho do projeto dos sintetizadores e respectivos osciladores de referência. Esse tipo de ruído é um parâmetro de grande importância em sistemas que utilizam modulação em fase [7].

Adicionalmente, mediu-se a BER para cada um dos canais,

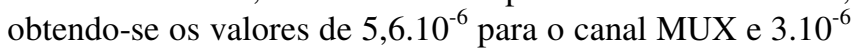
para o canal WFI. Esses valores foram medidos na condição de $\mathrm{Eb} / \mathrm{N} 0$ de 10,5 dB, que corresponde teoricamente à BER de $1.10^{-6}$, para a modulação em consideração. Ressalta-se que na condição real o sistema vai operar em uma situação muito mais favorável com relação a BER, uma vez que a degradação total medida encontra-se abaixo do valor total especificado $(6 \mathrm{~dB})$

\section{TESTES AMBIENTAIS}

O transmissor em questão foi projetado para servir como modelo de desenvolvimento do Subsistema MWT, do satélite CBERS $3 \& 4$.

Dentro do escopo do projeto MWT [2], o próximo passo será a fabricação do modelo de qualificação (MQ) do 
transmissor, o qual deverá ser submetido a uma bateria de testes [6] em um ambiente espacial simulado. Apresentam-se a seguir uma descrição dos testes de qualificação:

- Inspeção Visual / Inspeção de Dimensionamento / Propriedades de Massa.

- Teste Funcional Inicial

- $\quad$ Pré-aquecimento (Burn-In)

- $\quad$ Teste Funcional

- Teste Térmico / Teste Funcional

- $\quad$ Ciclagem Térmica / Teste Funcional

- $\quad$ Termo-Vácuo / Teste Funcional

- Vibração

- $\quad$ Teste Funcional Final

\section{Teste Funcional}

De acordo com os requisitos elétricos para este equipamento o teste funcional é composto de: Consumo DC, Freqüência de Saída, Potência RF de Saída, Espúrios e Harmônicos, Parâmetros de Modulação, Isolação entre Canais, Desbalanceamento de amplitude e fase, EVM e BER.

\section{Burn-In}

Este teste é realizado com o equipamento ligado para antecipar possíveis falhas prematuras nos componentes. $\mathrm{O}$ TX é mantido sobre aquecimento de $+55^{\circ} \mathrm{C}$ por 24 horas e após este período um teste funcional é realizado para verificação do desempenho.

\section{Teste Térmico}

A idéia deste teste é submeter o equipamento a temperaturas $10^{\circ} \mathrm{C}$ acima e abaixo do valor nominal de operação para garantir uma boa margem durante a operação normal.

Particularmente a esta missão o TX será submetido à faixa de temperatura de $-20{ }^{\circ} \mathrm{C}$ to $+55^{\circ} \mathrm{C}$ em passos de $10{ }^{\circ} \mathrm{C}$. Os testes funcionais são realizados em cada patamar temperatura.

\section{Ciclagem Térmica}

O equipamento deve ser submetido ao perfil térmico mostrado na Figura 7. Um teste funcional reduzido é realizado durante as temperaturas $\mathrm{T} 1, \mathrm{~T} 2, \mathrm{~T} 3, \mathrm{~T} 4, \mathrm{~T} 5$ e T6.

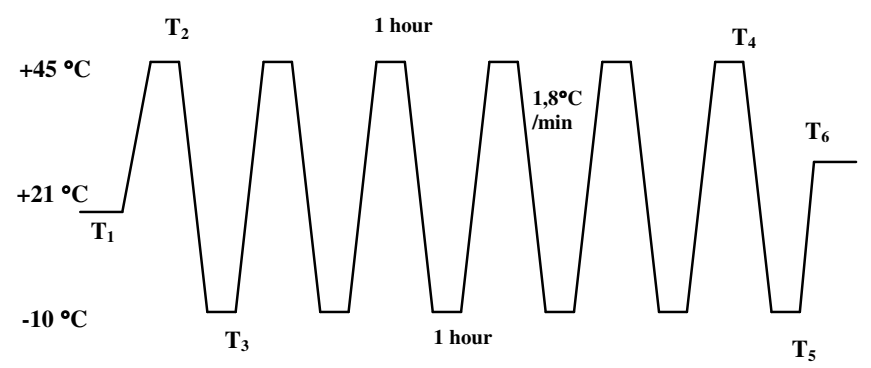

Fig. 7. Perfil da Ciclagem Térmica

\section{Termo - Vácuo}

Neste teste o equipamento é submetido à temperatura e pressão especificadas no documento "CBERS 3\&4 RBDCS-0001/02 - Design and Construction Specification" [9].

Um teste funcional é realizado nos patamares quente $\left(+45^{\circ} \mathrm{C}\right)$ e frio $\left(-10^{\circ} \mathrm{C}\right)$. O perfil deste teste é mostrado na Figura 8.

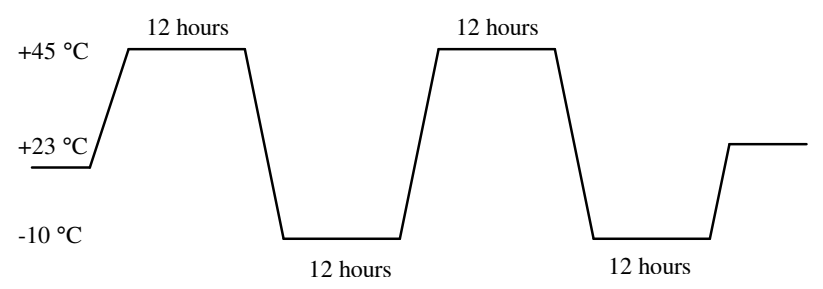

Fig. 8. Perfil do teste Termo-Vácuo

\section{CONCLUSÃO}

No projeto do TX foram utilizados componentes modulares disponíveis no mercado internacional, tendo por objetivo a otimização de massa, volume e consumo de potência, os quais são parâmetros fundamentais para equipamentos com aplicação espacial. Como já citado, este tipo de transmissor não é encontrado no mercado nacional e a sua aquisição no mercado internacional esta sujeita às restrições impostas pelo ITAR [3]. Além disso, deve-se ressaltar o elevado custo desse tipo de equipamento para uso em satélites, o que justifica o desenvolvimento desse tipo de transmissor no país.

Os resultados finais comprovam que o transmissor opera corretamente dentro das especificações do subsistema MWT [2]. O próximo passo será submeter o equipamento aos testes ambientais citados, no intuito de avaliar o seu desempenho em um ambiente espacial simulado.

\section{REFERÊNCIAS}

[1] CBERS 3\&4 - Chinese Brazilian Earth Resources Satellite http://www.cbers.inpe.br/

[2] R. Araujo, L. Cividanes, MUX \& WFI Transmitter (MWT) Subsystem Specification - RBK-HDS-0011/03 - INPE - 2004.

[3] ITAR, http://pmddtc.state.gov/itar index.htm (acesso em 30/04/2007)

[4] MITEQ, http://www.miteq.com (acesso em 30/04/2007)

[5] G. Maral e M. Bousquet, Satellite Communication, Wiley, 1993

[6] ZODIAC - IN-SNEC - CORTEX Series - High Data Receiver manual.

[7] R. Gagliard, Satellite Communication System, VNR, 2002.

[8] R. Araujo, I. Tosetto, J. Rae, J. A. Rodrigues and C. Gonçalves. Procedimento de Testes do Transmissor SATEC, doc. SATEC-TSP-001, INPE - São José dos Campos, SP, 2002.

[9] "CBERS 3\&4 RB-DCS-0001/02 - Design and Construction Specification". INPE, 2005. 